Dentists cannot (in my view) abdicate the provision of oral hygiene to the rest of the dental team.

\section{Twice a day?}

For as long as I can remember the standard advice for the prevention of both dental caries and periodontal disease was to brush your teeth twice a day. There is no doubt this message has reached the general public, even if not everyone takes notice of it, and most people involved in oral healthcare continue to broadcast this message as often as possible. In fact, brushing your teeth twice a day forms the main theme of the Colgate/BDA Oral Health Month which is taking place in the UK throughout September this year.

At first glance you might feel (as I confess I did) that this theme of brushing your teeth is possibly both obvious and a little condescending to patients who keep hearing this message all the time. After all, surely everyone knows by now that dentists, hygienists, oral health educators and everyone involved in health care all preach the message of twice-daily brushing? We appreciate not everyone chooses to take notice of our message, but at least we have 'done our bit' and told them they should, and if they choose to ignore our advice then so be it. We cannot force people to brush regularly, nor should we. Our responsibility ends with the advice.

But if we do feel this theme is too obvious then are we missing something? Perhaps we feel that this message has become so successful in the past that we forget how important it still is. Perhaps we have assumed that we have been saying it for so long that we no longer need to. Perhaps the more innovative and exciting areas of dental care (such as implants, tooth whitening and advanced restorations) have pushed aside the real basics of effective dentistry - basic oral hygiene.

All I have written so far acts as a perfect introduction to the paper in this issue on toothbrushing, the fourth paper in the series on prevention. The authors have looked at the research to support the advice on toothbrushing, grading that evidence and revealing that although past research to support the benefits of regular plaque control may be a little weak by today's standards, there are an increasing number of studies that do support the benefit of regular toothbrushing in the prevention of caries and periodontal disease.
We really do need this stronger evidence, because messages for regular toothbrushing in the prevention of caries have occasionally been contradictory in the past, resulting in confusion in the minds of both patients and dental educators. Should we brush before or after breakfast? Does toothbrushing prevent caries at all? How important is brushing compared with diet control? What type of brush works? Do electric toothbrushes really make a difference? Some of the answers to these questions are emerging, and this paper not only provides current thinking but also grades the evidence for that current thinking as well. Knowing the scientific strength of evidence is extremely useful, and I hope we see more and more of this in the future.

The paper not only clarifies current thinking on the background and support for the effect of regular toothbrushing on caries, but also looks at how plaque control effects periodontal disease. This is an area where I have always felt more confident about providing advice to patients, and I was heartened to see things seem to be the same, although the evidence is still apparently not scientifically outstanding.

Finally, the paper deals with the very subject I mentioned at the beginning of this leader - the importance of the continuing role the clinician plays in providing appropriate advice on toothbrushing for patients. The Colgate/BDA Oral Health Month reminds us that our involvement, whether we are general practitioners, specialists, hospital or community practitioners, remains paramount. Dentists cannot (in my view) abdicate the provision of oral hygiene to the rest of the dental team. Everyone in the practice must continue to stress its importance, either by backing up advice given by oral health educators, hygienists and therapists, or by remembering to repeat the message when contemplating treatment plans. It may seem to us to be obvious, but our personal involvement is not only required but essential. 